\title{
Neutron, fluorescence, and optical imaging: An in situ combination of complementary techniques
}

\author{
D. Wagner, ${ }^{1}$ M. Börgardts, ${ }^{2}$ C. Grünzweig, ${ }^{3}$ E. Lehmann, ${ }^{3}$ T. J. J. Müller, ${ }^{2}$ S. U. Egelhaaf, ${ }^{1}$ \\ and H. E. Hermes ${ }^{1}$ \\ ${ }^{1}$ Condensed Matter Physics Laboratory, Heinrich Heine University, 40225 Düsseldorf, Germany \\ ${ }^{2}$ Institute for Organic and Macromolecular Chemistry, Heinrich Heine University, 40225 Düsseldorf, Germany \\ ${ }^{3}$ Neutron Imaging and Activation Group, Paul Scherrer Institute, 5232 Villigen, Switzerland
}

(Received 10 July 2015; accepted 8 September 2015; published online 23 September 2015)

\begin{abstract}
An apparatus which enables the simultaneous combination of three complementary imaging techniques, optical imaging, fluorescence imaging, and neutron radiography, is presented. While each individual technique can provide information on certain aspects of the sample and their time evolution, a combination of the three techniques in one setup provides a more complete and consistent data set. The setup can be used in transmission and reflection modes and thus with optically transparent as well as opaque samples. Its capabilities are illustrated with two examples. A polymer hydrogel represents a transparent sample and the diffusion of fluorescent particles into and through this polymer matrix is followed. In reflection mode, the absorption of solvent by a nile red-functionalized mesoporous silica powder and the corresponding change in fluorescent signal are studied. () 2015 AIP Publishing LLC. [http://dx.doi.org/10.1063/1.4931427]
\end{abstract}

\section{INTRODUCTION}

Interest in soft matter and material sciences moves towards increasingly complex samples, e.g., host-guest systems or composite materials. ${ }^{1-4}$ In addition, these are often studied under rather complicated conditions, e.g., external forces, such as shear, ${ }^{5-7}$ or whilst undergoing structural or phase transitions. ${ }^{8-11}$ Furthermore, biologically or industrially relevant samples are typically multi-component systems. ${ }^{12}$ Due to their complex nature, completely reproducible samples and kinetic responses are not usually observed such that sequential measurements have only limited value and a better understanding of the samples' behavior can often be obtained by applying different complementary measurement techniques, at the same time. ${ }^{13,14}$ In this article, we describe a setup that has been designed to perform three complementary imaging measurements, namely, optical imaging (OI), fluorescence imaging (FI), and neutron radiography (NR), simultaneously, i.e., at the same time and on the same sample volume.

A large variety of imaging methods are used in soft matter science, biology, and medical diagnostics. ${ }^{15,16}$ Their basic principle is similar: radiation interacts with the sample and the resulting signal is recorded with a two-dimensional detector leading to an image that contains spatially resolved information about certain characteristics of the object. Different features of the sample can be "imaged," depending on the chosen technique and contrast.

In OI, the transmission or reflection of visible light by the sample is recorded as a function of space and time. ${ }^{17}$ The recorded light intensity relative to the incident intensity reflects the local optical density of the sample along the light path. By contrast, FI provides information on the local concentration of fluorophores. ${ }^{18}$ The fluorophores are excited by the incident radiation and emit light with a longer wavelength. The excitation and emission spectra depend on the specific fluorophore and determine the choice of the illumination wavelength(s) and the spectral characteristics of the filters used in the imaging part of the device. The fluorophore is chosen to report on the feature of interest, such as the motion of the fluorophore itself, a local property or the local concentration of some chemical. ${ }^{19}$ The fluorophore is thus a specific marker which usually has to be added to the system and hence potentially changes the system. NR provides a transmission image, which depends on the neutron attenuation of the sample and thus probes a different aspect of the sample compared to OI and FI. Neutrons interact with the nuclei and hence the interaction depends on the specific isotope. Therefore, isotope exchange can be exploited to highlight specific parts of the sample, similar to the addition of fluorophores in FI except that isotope exchange tends to affect the sample less. In studies involving biological or soft matter samples, typically the large difference in the attenuation of hydrogen and deuterium is exploited and deuteration is used to highlight or mask specific parts of the sample. ${ }^{20}$ Due to the much smaller wavelength of neutrons compared to light, the scattering of neutrons by large-scale structures, such as those of interest in biological or soft matter samples, occurs at much smaller scattering angles. Thus, for spatial resolutions and sample thicknesses similar to the ones in these experiments, neutrons scattered by largescale structures are detected by the same or a neighboring pixel as the transmitted beam. The observed effective transmission is hardly reduced by scattering effects which can hence often be neglected in the data analysis. ${ }^{21-23}$

Neutron radiography and fluorescence imaging have been combined before ${ }^{19,24}$ In these previous studies, however, the samples had to be moved out of the neutron beam to take fluorescence images. Thus, a truly simultaneous observation as well as an imaging of fast kinetic processes was not possible. A further advantage of the setup described here is the 
significantly better spatial resolution, which is crucial for typical biological or soft matter samples.

\section{EXPERIMENTAL SETUP}

The setup that combines optical, fluorescence, and neutron imaging can be operated in two modes: optical transmission and optical reflection imaging. This allows the investigation of optically transparent and opaque samples. The optical imaging setup is built on an optical breadboard $(45 \mathrm{~cm} \times 60 \mathrm{~cm})$ which is placed in the neutron beamline and aligned to the neutron beam. In both setups, neutron radiography is performed in transmission mode.

\section{A. Neutron, fluorescence, and optical imaging in transmission mode}

The imaging setup in transmission mode is schematically shown in Figure 1(a) and photographs are presented in Figure 2. The setup is designed so that, apart from the sample

(a) Optical Transmission Mode

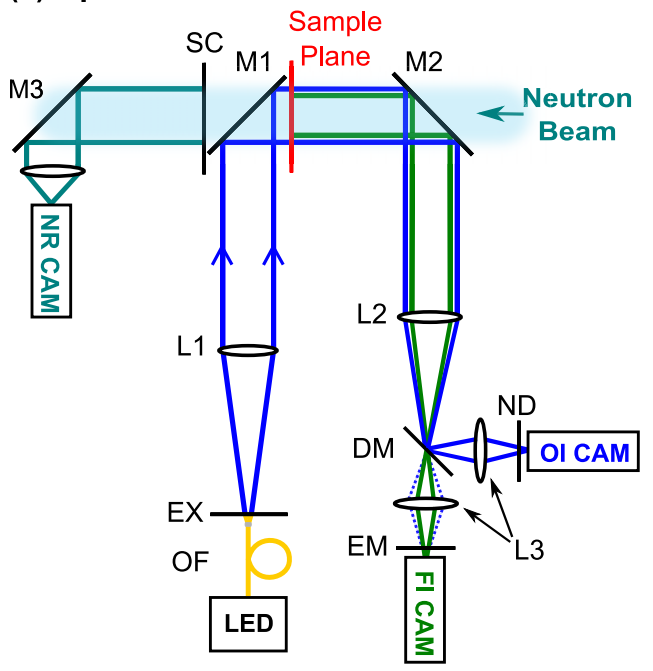

(b) Optical Reflection Mode

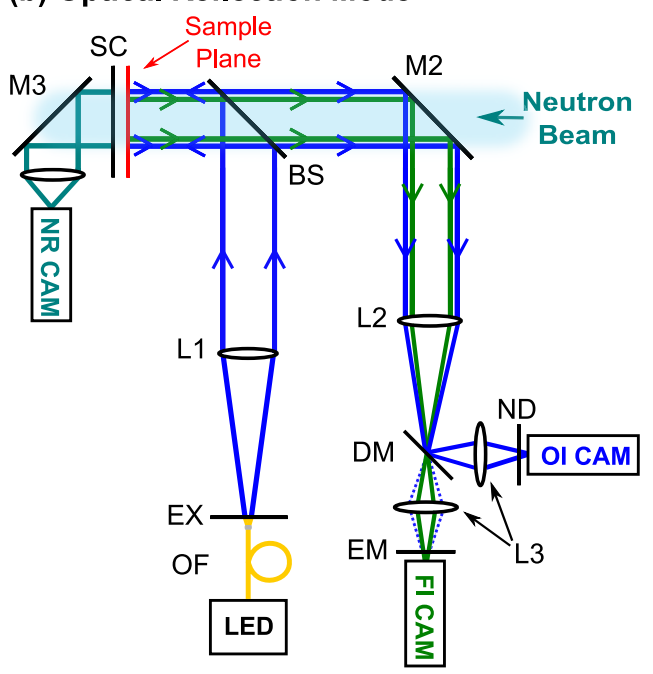

FIG. 1. Schematic representation of the experimental setup in (a) optical transmission and (b) optical reflection modes. See text for details.
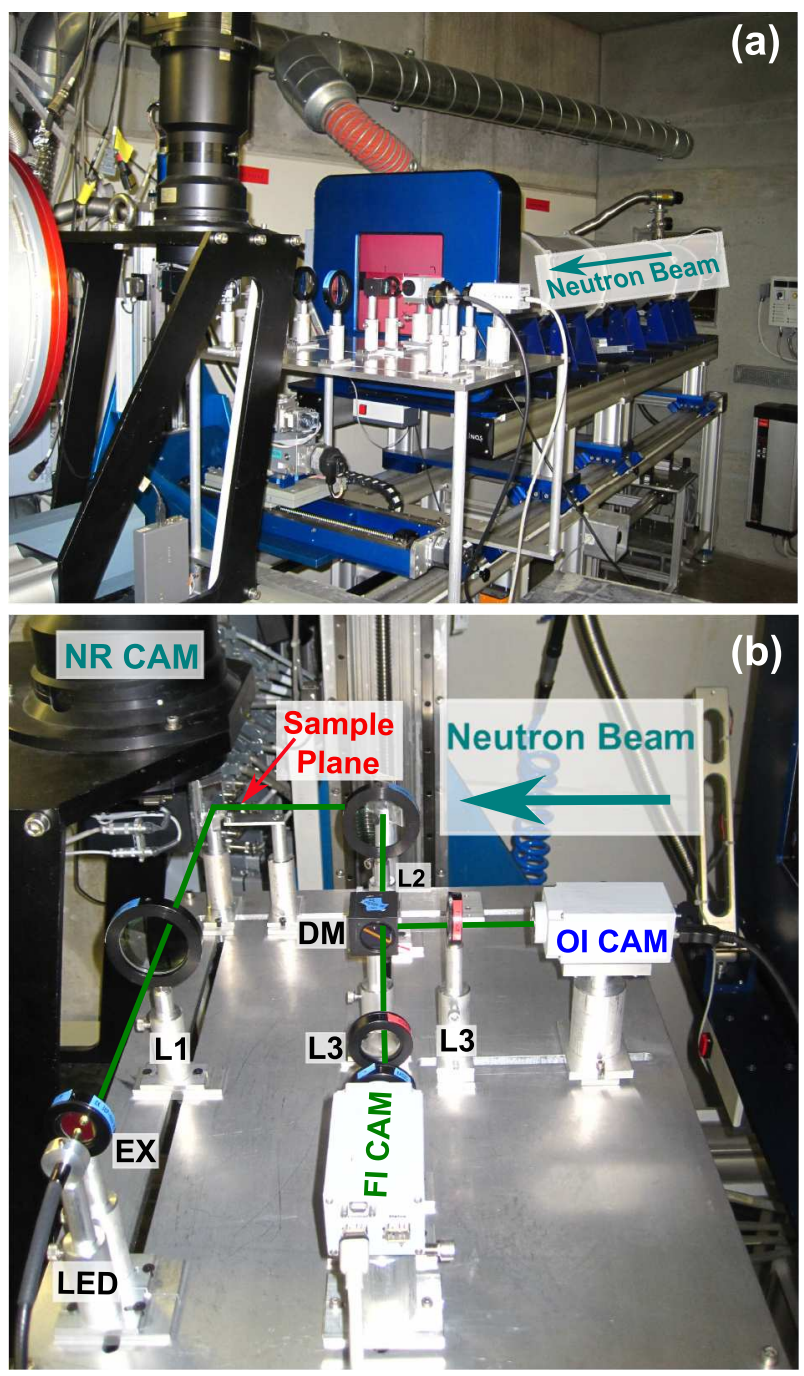

FIG. 2. Optical and fluorescence imaging setup in transmission mode in the neutron radiography beamline ICON at the Paul Scherrer Institute. The labels are as in Figure 1 and defined in the main text. Note that there is no sample cell in the sample plane and shielding to protect the equipment, especially the cameras, from radiation damage, has been removed.

cell, only two optical mirrors (M1 and M2) are located in the neutron beam. The two mirrors are made of quartz glass with a chrome-silica coating and they are sufficiently transparent to neutrons so that the neutron beam is not significantly attenuated or otherwise affected. Nevertheless, the mirrors are accounted for in the data analysis by applying a bright beam correction (Sec. III A). The other components and especially the CCD cameras are placed far enough from the neutron beam and are shielded to minimize their activation. The sample is placed in the neutron beam, as close as possible in front of the scintillator (see below). The transmitted neutrons reach the scintillation screen (SC) which then emits photons. A $10 \mu \mathrm{m}$ thick gadolinium based scintillator $\left(\mathrm{Gd}_{2} \mathrm{SO}_{2} \mathrm{~S}\right.$ : $\left.\mathrm{Tb}\right)$ has been found to be suitable for high-resolution neutron imaging. The emitted photons are reflected from mirror M3 and recorded with a CCD camera (NR cam) ${ }^{25}$ Thus, the gray value in each pixel of the image provides information on the transmitted neutron intensity in the corresponding sample volume.

The divergence of the neutron beam limits the resolution of the image to ${ }^{26}$ 


$$
u_{\mathrm{g}}=\frac{l}{L / D_{\mathrm{ap}}},
$$

where $l$ is the sample-scintillator distance, $D_{\text {ap }}$ is the diameter of the source aperture and $L$ is the distance of this aperture to the detector, i.e., the scintillator. While $L$ and $D_{\text {ap }}$ are set by the neutron beamline, the sample-scintillator distance $l$ should be minimized in order to reduce geometrical blurring. In this setup, $l$ is limited by the sizes of the sample cell (Sec. II C) and the mirror M1 which is why this mirror is kept as small as possible while still covering the whole observation volume.

An LED lamp (CoolLED pE-2) is chosen as light source for the optical and fluorescence parts of the apparatus. Its intensity was found to be sufficient for our samples and stable for long times. Importantly, its wavelength can be adapted to the excitation spectrum of the fluorescent dye molecules in the sample. This LED lamp can also provide several wavelengths which can be used to excite different fluorophores simultaneously. The light from the LED lamp is guided by an optical fiber (OF) to an excitation filter (EX). This is a short pass or bandpass filter that transmits a narrow range of wavelengths. It ensures that the wavelength of the incident light is well below the emission wavelength of the fluorophore. The light is then collimated using an achromatic doublet lens with focal length $f_{1}=20 \mathrm{~cm}(\mathrm{~L} 1)$. The lens is placed at its focal length from the exit of the fiber and hence, the sample is illuminated by a parallel beam of light. Compared to a focused illumination beam, this exposes the dye molecules to a significantly lower intensity and thus reduces photobleaching. To increase the illumination intensity, a condenser lens could be added behind the optical fiber in order to collect light from a larger solid angle. This was not necessary for our samples.

Using a dichroic mirror (DM), the light transmitted by the sample is split into two parts. First, the short-wavelength part, which is the transmitted excitation light that forms the optical transmission image. Second, the long-wavelength part, namely, the light emitted by the fluorophores which forms the fluorescence image. These two parts are separately recorded using two cameras (OI cam and FI cam) for which we use one black and white and one color CCD camera (AVT Stingray), respectively. To reduce the transmitted intensity if necessary, a neutral density filter (ND) can be introduced in front of the OI cam. To ensure that only light emitted by the fluorophores is recorded, an emission filter (EM) is added directly in front of the FI cam. The two images are formed using lenses (L2 and a pair L3). The magnification is determined by the relative focal lengths and positions of the objective lens, an achromatic doublet with focal length $f_{2}=20 \mathrm{~cm}$ (L2), and the focusing lenses, achromatic doublets with $f_{3}=7.5 \mathrm{~cm}$ (L3). In the experiments described here, we typically observe an area of about $2 \mathrm{~cm} \times 2 \mathrm{~cm}$. This exceeds the sensor size of the cameras $(0.85 \mathrm{~cm} \times 0.71 \mathrm{~cm})$ and thus a magnification smaller one is necessary; this is realized by choosing focusing lenses (L3) with a focal length smaller than that of the objective lens (L2), $f_{3}<f_{2}$.

The use of a color camera as the FI cam allows changes in the emission spectrum during an experiment to be investigated. Depending on the emitted wavelength, the analysis can be restricted to only one channel of the color camera to enhance the signal-to-noise ratio. The OI camera together with an additional emission filter could also be used to monitor a second fluorophore with a different emission spectrum.

\section{B. Fluorescence and optical imaging in reflection mode and neutron imaging in transmission mode}

The investigation of samples that are opaque to light but transparent to neutrons is possible after small modifications to the setup. As shown in Figure 1(b), epi-illumination is realized by using a beam splitter (BS) instead of the mirror M1. A beam splitter that transmits $70 \%$ and reflects $30 \%$ of the incident light was found to work well. The beam splitter directs the incident light onto the sample. The reflected as well as the emitted light travel back in the direction of the incident beam and through the beam splitter. As in transmission mode, both parts are separated by the DM and imaged by lenses (L2 and L3) onto two separate cameras (OI cam and FI cam). Since mirror M1 is no longer needed, the sample can be moved closer to the scintillator, thus decreasing the geometrical blurring $u_{\mathrm{g}}$.

\section{Sample cells}

In order to be able to analyze the data on a quantitative level, the sample cell has to meet some requirements. Its transmission has to be high for neutrons, and for light, at least one transparent window is required. Suitable materials for neutron radiography are, e.g., aluminum or quartz glass. The latter is also transparent to visible light and thus the material of choice for combined imaging experiments. To determine the sample composition from its transmission, a uniform and precisely known sample thickness is required. Furthermore, as already mentioned, it is also desirable to minimize the sample-scintillator distance. Thus, the side of the sample cell pointing towards the scintillator should be kept as thin as possible. Due to the horizontal neutron beam, the cell has to be constructed such that a vertical sample orientation is possible. Depending on the sample and experiment to be performed, it can be desirable to construct cells which are demountable and/or watertight. Temperature or pressure control is another feature that might be considered.

For the transmission experiments described below (Sec. IV A), demountable and watertight sample cells were designed (Fig. 3). They consist of a two-piece aluminum alloy body. In contrast to a previous cell consisting only of aluminum, ${ }^{27}$ the present cell has quartz glass windows. One aluminum part has a spherical window (Hellma, $22 \mathrm{~mm}$ diameter, $1.25 \mathrm{~mm}$ thickness) glued to it. The sample is placed on this window and then a second quartz glass window is put on top of the sample, whose thickness is determined by a fixed aluminum spacer ring of $0.5 \mathrm{~mm}$ thickness and, if required, additional aluminum spacer rings. The cell is sealed using an O-ring and the second aluminum part, which is screwed to the cell body with anodized aluminum screws. The inside of the aluminum body is covered with an inert anti-reflection foil (not shown in Fig. 3) to prevent reflections from the inner walls of the sample cell which can be caused by a slight misalignment of the sample cell with respect to the light path and can lead to, e.g., a bright spot in the center of the cell due to its cylindrical 

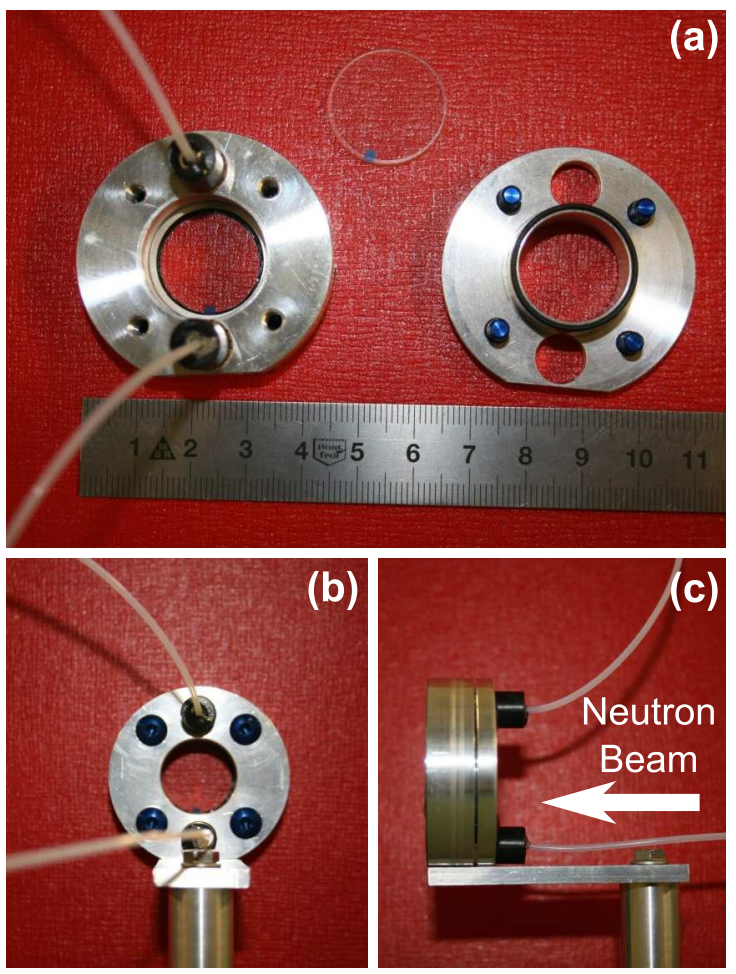

FIG. 3. Demountable watertight sample cell with quartz glass windows and a cell body made of aluminum: demounted cell (a), front (b), and side view (c) of the cell.

geometry. The sample cell has two holes that are connected to polytetrafluoroethylene (PTFE) tubes. Through the tubes, the mounted cell can be degassed and filled with liquids. This allows the sample to be monitored in situ before and during contact with a liquid.

Although this cell can also be used in reflection mode, it is not suitable for the second set of experiments presented (Sec. IV B). Thus, a different cell was designed (Fig. 4). It consists of a single aluminum body with one solvent chamber, two rectangular sample chambers, and a front window made of a glass slide with a thickness of $1.05 \mathrm{~mm}$. The sample chambers have a height of about $30 \mathrm{~mm}$ and a square cross section of $5.1 \mathrm{~mm} \times 5.1 \mathrm{~mm}$, i.e., a path length of $5.1 \mathrm{~mm}$

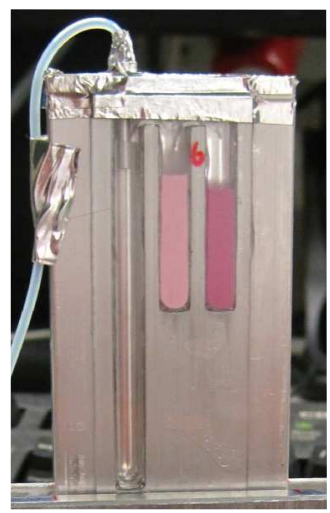

FIG. 4. Sample cell with two sample chambers filled with dye-functionalized mesoporous silica (middle and right) and one solvent chamber (left). The chambers are connected at the top by a groove. The cell is closed with an aluminum lid that is sealed with aluminum adhesive tape which, in the photograph, covers the groove. while the solvent chamber is taller. All chambers are connected at the top such that saturated solvent vapor is in contact with the samples. The solvent chamber can be filled through PTFE tubes after the cell has been mounted in the neutron beam.

\section{DATA TREATMENT}

The raw data obtained from the experiments are stored as series of grayscale or (one channel of) color images depending on the camera used. The time series of two-dimensional images are recorded as intensities of every pixel, $I_{\text {raw }}(x, y, t)$, and are subsequently analyzed.

\section{A. Neutron radiography}

The measured time series of images, i.e., two-dimensional intensities $I_{\text {raw }}(x, y, t)$, are reduced and analyzed as described elsewhere ${ }^{28}$ and briefly summarized here. (For clarity, in the following, the dependence on the position of the pixel, $(x, y)$, and the time since the experiment has been started, $t$, is not explicitly given.)

To correct for effects caused by spatial variations in the beam intensity or in the detector efficiency as well as background and electronic noise, a pixelwise correction is applied to $I_{\text {raw }}$ to yield the transmission, $T$,

$$
T=\frac{I_{\mathrm{raw}}-I_{\mathrm{D}}}{I_{\mathrm{B}}-I_{\mathrm{D}}},
$$

where $I_{\mathrm{D}}$ is the intensity measured with all shutters closed ("dark image"). Furthermore, $I_{\mathrm{B}}$ denotes the intensity of a "bright image," taken without a sample but with the optical setup, in particular mirror M2 and mirror M1 or the BS, in the neutron beam.

Even though the NR camera is not directly irradiated by the neutron beam, its CCD chip is exposed to some radiation that results in sporadic high local intensities in the image ("gamma spots"). For $I_{\mathrm{D}}$ and $I_{\mathrm{B}}$, a median filter on a stack of ten images is used to avoid effects of gamma spots. ${ }^{29}$ This approach is not useful for radiographs that capture dynamic processes and is thus not applied to our sample images $I_{\text {raw }}$. For these images, the affected pixels are not included in the data analysis. Figure 5 shows a neutron radiograph of a polymer
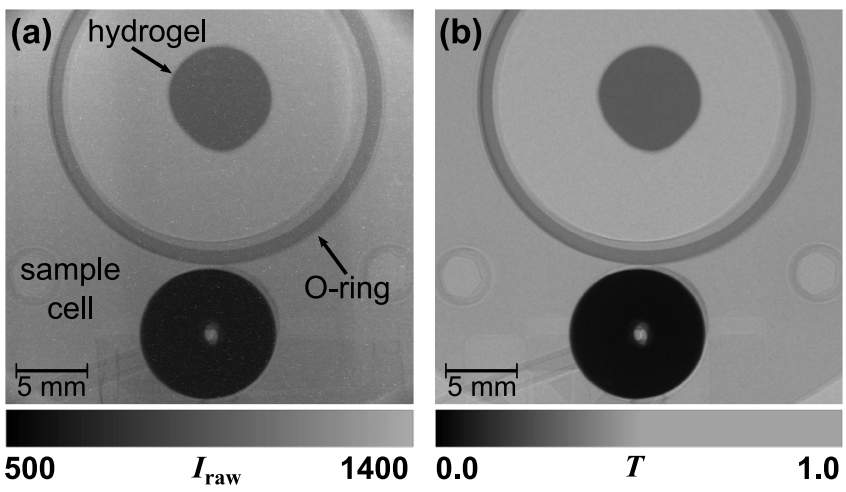

FIG. 5. Neutron radiograph of a polymer hydrogel disc and parts of the sample cell (a) before and (b) after a correction for beam and detector inhomogeneities, background noise and gamma spots, i.e., (a) represents the recorded intensity $I_{\text {raw }}$ and (b) the transmission $T$ (Eq. (2)), respectively. 
hydrogel inside the sample cell before and after application of the corrections described by Eq. (2), which illustrates that the image becomes more homogeneous by the correction.

The neutron beam might not only be spatially inhomogeneous but often also fluctuates with time $t$. Therefore, for time series, the transmission is normalized by the actual incident neutron intensity or a substitute of this quantity that shows the same time dependence. The mean intensity in a reference area where no changes are expected throughout the measurement (for instance, a part of the cell body) can be used. Similar to Eq. (2), the transmission, averaged over this reference area, relative to the incident beam is

$$
\left\langle T_{\text {ref }}\right\rangle=\frac{\left\langle I_{\text {raw,ref }}\right\rangle-\left\langle I_{\mathrm{D}, \text { ref }}\right\rangle}{\left\langle I_{\mathrm{B}, \text { ref }}\right\rangle-\left\langle I_{\mathrm{D}, \text { ref }}\right\rangle},
$$

where the intensities of the raw, dark, and bright images are averaged over the same pixels, representing the reference area. This allows us to calculate the transmission relative to the reference area and hence correct for time-variations in the incident intensity,

$$
T_{\text {rel }}=\frac{T}{\left\langle T_{\text {ref }}\right\rangle} .
$$

We can now calculate the transmission of the sample, $T_{\mathrm{S}}$, i.e., the transmission of the sample in the cell, $T_{\text {rel,SC}}$, without the contribution of the transmission of the empty cell, $T_{\mathrm{rel}, \mathrm{C}}$,

$$
T_{\mathrm{S}}=\frac{T_{\mathrm{rel}, \mathrm{SC}}}{T_{\mathrm{rel}, \mathrm{C}}} .
$$

Note that the values of $\left\langle T_{\text {ref }}\right\rangle$ for sample and empty cell are not necessarily the same as they are obtained at different times and thus $T_{\mathrm{S}}$ is not necessarily $T_{\mathrm{SC}} / T_{\mathrm{C}}$.

According to the Lambert-Beer law, the transmission of radiation traveling through the sample is linked to the materialspecific attenuation coefficient $S$ and the path length $d$,

$$
T_{\mathrm{S}}=e^{-S d} .
$$

For a one-component material $i, S_{i}$ is related to the number $n_{i, j}$ of each kind of nucleus $j$ in the material (or constituting molecule) $i$ and the total neutron cross section of that nucleus, $\sigma_{j},{ }^{30,31}$ according to

$$
S_{i}=\frac{\rho_{i}}{M_{i}} N_{\mathrm{A}} \sum_{j} n_{i, j} \sigma_{j},
$$

where $\rho_{i}$ is the density, $M_{i}$ the molar mass of the molecule, and $N_{\mathrm{A}}$ Avogadro's number. The attenuation coefficients $S_{i}$ can hence be calculated or determined experimentally. For a sample consisting of different components with volume fractions $\phi_{i}$, the total attenuation coefficient is

$$
S=\sum_{i} \phi_{i} S_{i}
$$

For two-component samples, using Eqs. (6) and (8), the local volume fractions $\phi_{i}(x, y, t)$ of the two components can be extracted from the images, provided that the sample thickness $d$ and the attenuation coefficients $S_{i}$ are known.

\section{B. Optical and fluorescence imaging}

It was found that the optical illumination and detection are homogeneous and stable in time, and the intensity is so high that the background is negligible and the used sample cells are sufficiently transparent to light that corrections as in Eqs. (2), (4), and (5) are not necessary for the optical and fluorescence images, i.e., $T_{\mathrm{s}}=I_{\text {raw }} / I_{\mathrm{B}} \propto I_{\text {raw }}$. However, as in any fluorescence experiment, the intensity might be affected by photobleaching. If the fluorescence intensity decreases in a reference area where no change in composition and hence intensity is expected, a photobleaching correction should be applied. ${ }^{32,33}$ If photobleaching is taken into account, we found, for the fluorescent dyes and their concentrations used in the present experiments, a linear relation between corrected intensity and dye concentration, i.e., a linear expansion of Eq. (6), can be used. Thus, the volume fraction of the fluorescent dye is directly related to the intensity $I_{\text {raw }}$.

\section{EXAMPLES}

The capabilities of the setup are illustrated by two examples. The first experiment used the transmission mode to simultaneously investigate the diffusion of dye molecules into a water-swollen polymer hydrogel, the diffusion of solvent molecules into and out of the hydrogel, and the swelling of the hydrogel. In the second example, dye-functionalized mesoporous silica was contacted with solvent vapor and, using the reflection mode, the change in fluorescence was compared with the local solvent concentration.

The experiments were performed at the cold neutron imaging facility ICON at the Paul Scherrer Institute (Villigen, Switzerland). ${ }^{34,35}$ The chosen distance of the source aperture, $L$, and diameter of the aperture, $D_{\text {ap }}$, imply $L / D_{\text {ap }}=343$. For the samples investigated, this represents the optimum compromise between neutron beam intensity and spatial resolution, which, for the specific sample position, i.e., $l$, was better than $100 \mu \mathrm{m}$ in all measurements. Images were collected continuously with an individual measurement time of $20 \mathrm{~s}-30 \mathrm{~s}$. Better spatial resolution or much shorter measurement times are possible if appropriate.

Optical transmission and fluorescence images were collected using a camera resolution of $10 \mu \mathrm{m}$, a field of view of about $25 \mathrm{~mm} \times 21 \mathrm{~mm}$, and a spatial resolution in the sample plane of about $20 \mu \mathrm{m}$. The resolution was chosen to roughly match that of the corresponding neutron radiography measurements. Again, a much better spatial resolution can be obtained if required.

\section{A. Transmission mode: Dye and solvent diffusion into and out of a polymer hydrogel}

The aim of this experiment was to investigate the diffusion of fluorescent dye into a polymer hydrogel and compare it to that of the solvent while also monitoring the swelling of the hydrogel. Since the sample is transparent to visible light, the setup was used in transmission mode (Fig. 1(a)). This experiment allowed us to separately but simultaneously follow solvent and solute movement with NR and FI, respectively. At 
the same time, the hydrogel was observed with OI to quantify its swelling or its changing appearance, for example, the development or healing of defects.

Polyacrylamide (PAAm) hydrogels with a cross-linker to monomer ratio of 1:60 were synthesized in light water $\left(\mathrm{H}_{2} \mathrm{O}\right)$ following a standard protocol. ${ }^{36,37}$ The initial water content of the hydrogel can be varied by partially drying of the hydrogel; it shrinks upon drying and re-swells after being contacted with solvent. Here, hydrogel discs with a light water $\left(\mathrm{H}_{2} \mathrm{O}\right)$ volume fraction of $\phi_{\mathrm{H}_{2} \mathrm{O}}=0.97$, radius $r \approx 4.5 \mathrm{~mm}$, and height $h$ $\approx 1.5 \mathrm{~mm}$ were cut from the hydrogel and transferred into the sample cell. By using additional spacers in the cell (Sec. II C), the sample was restrained to a thickness of $1 \mathrm{~mm}$ to prevent slipping due to the vertical sample orientation. The cell was then placed on its holder in the setup and aligned with respect to the scintillator. During installation of the optical setup, it had been aligned with the neutron beam, namely, the scintillator, so that an alignment of the cell with respect to the scintillator assures its proper alignment with the neutron and optical beams.

Fluorescein sodium salt (in the following simply referred to as fluorescein) was chosen as fluorescent dye. This was dissolved in heavy water $\left(\mathrm{D}_{2} \mathrm{O}\right)$ to obtain a concentration of $26.6 \mu \mathrm{M}$. Fluorescein is excited at $496 \mathrm{~nm}$ and emits at $525 \mathrm{~nm}$. Thus, an LED with a center wavelength of $490 \mathrm{~nm}$ and an EX filter that transmits a band of $29 \mathrm{~nm}$ width around a center wavelength of $480 \mathrm{~nm}$ were used. The emitted fluorescence and the excitation light were separated using a DM that transmits wavelengths above and reflects wavelengths below $502 \mathrm{~nm}$. Additionally, an EM filter that transmits only wavelengths above $520 \mathrm{~nm}$ was added.

At the start of an experiment, several neutron, fluorescence, and optical transmission images were collected before the hydrogel was contacted with dye solution. The fluorescein solution was injected using a syringe pump that could be controlled from outside the bunker of the beam line. Thus, images could be taken throughout the filling process. At the end of the filling process, the fluorescein solution filled the whole sample cell, hence completely surrounding the hydrogel. Since the faces of the disc were covered by the glass, diffusion of solvent and fluorescent particles only occurred through the circular edge, hence representing a quasi-twodimensional geometry of an infinite cylinder. Examples of neutron radiographs, fluorescence, and optical images taken immediately after contact with the fluorescein solution and 20 min later are shown in Fig. 6. Neutron imaging is sensitive to the isotopic composition of the sample (Secs. I and III A) and reveals the diffusion of heavy water (initially outside the hydrogel) and light water (initially in the hydrogel) into and out of the hydrogel, respectively. Initially, a strong contrast between the hydrogel and the surrounding solution is observed (Fig. 6(a), left). In the course of the experiment, the contrast between the hydrogel and the solvent becomes less pronounced (Fig. 6(a), right) and eventually vanishes. This indicates the inter-diffusion of heavy water and light water.

Fluorescence imaging shows an increasing fluorescence intensity and hence fluorescein concentration inside the hydrogel with time (Fig. 6(b)). This reflects the diffusion of fluorescein molecules into the hydrogel. The fluorescein molecules clearly enter the hydrogel at a slower rate than the heavy water. (a) Neutron Radiography

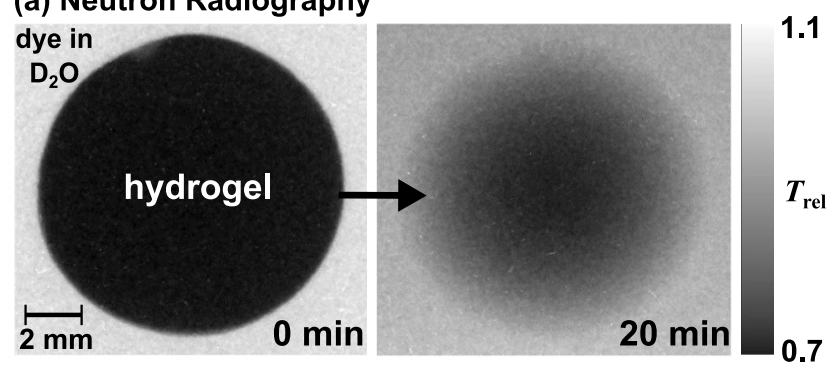

(b) Fluorescence Imaaing

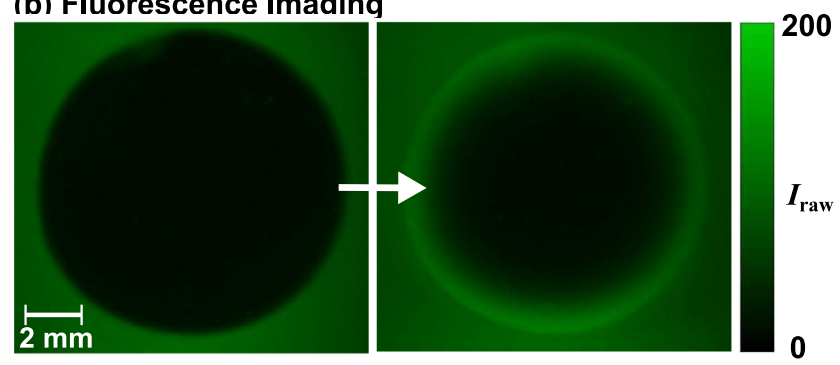

(c) Optical Imaging

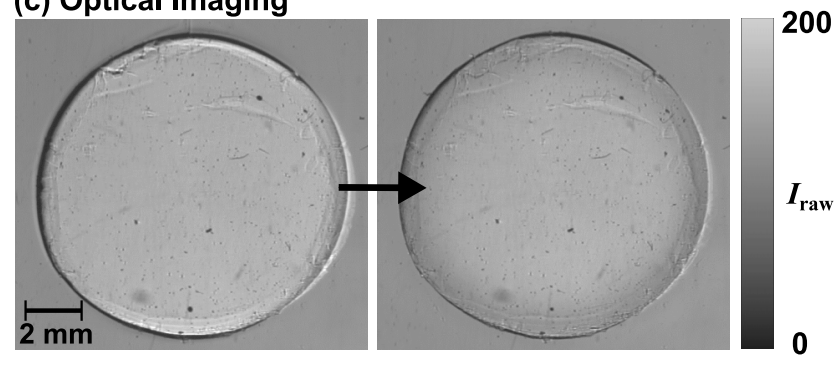

FIG. 6. Fully swollen polymer hydrogel in light water $\left(\mathrm{H}_{2} \mathrm{O}\right)$ (left) immediately and (right) $20 \mathrm{~min}$ after contact with fluorescein in heavy water $\left(\mathrm{D}_{2} \mathrm{O}\right)$ as observed simultaneously by (a) neutron radiography, (b) fluorescence imaging, and (c) optical transmission imaging.

This is consistent with the larger size, and thus a lower diffusion coefficient, of a fluorescein molecule compared to a water molecule. It also shows that the setup is able to discriminate between solvent and solute movements. Note that the bright rim at the hydrogel-solution interface arises from scattering at the (rough) hydrogel edge and precludes an analysis of the fluorescence data close to the interface. This effect is particularly pronounced, for example, compared to optical transmission imaging, as the fluorescence intensity is relatively low. It illustrates the advantage of neutrons with their smaller wavelength and thus smaller scattering angles such that neutrons scattered by mesoscopic structures are recorded by (almost) the same pixel as the transmitted neutrons. Furthermore, the refractive index differences experienced by neutrons are typically much smaller.

Optical transmission imaging shows a slight decrease in hydrogel size of about $0.03 \mathrm{~mm}$. The optical images also provide useful information on, e.g., defects, as can be seen in the upper left region of the hydrogel shown in Fig. 6(c). The evolution of the defects, for example, their healing, can be followed. Furthermore, since such defects lead to a faster ingress of the fluorescein solution, this region can be excluded from the quantitative analysis of the fluorescence and neutron images.

To access quantitative information from the images, the intensity, $I_{\text {raw }}$, was analyzed pixelwise (Sec. III) and then averaged to obtain the time-evolution of the concentration profiles, 
(a) Neutron Radiography

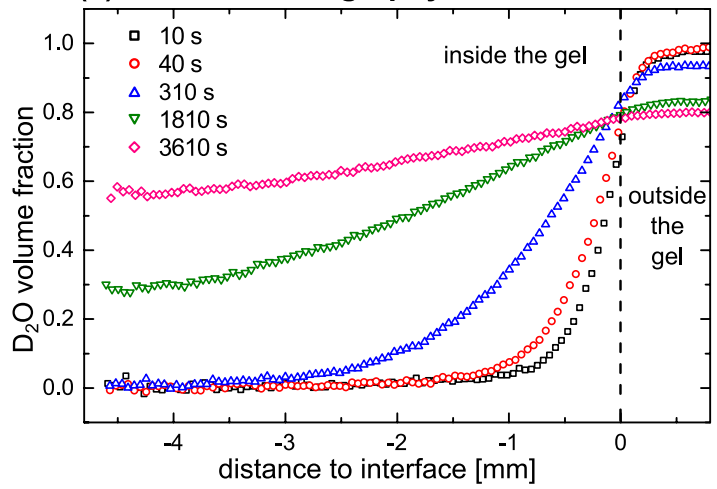

(b) Fluorescence Imaging

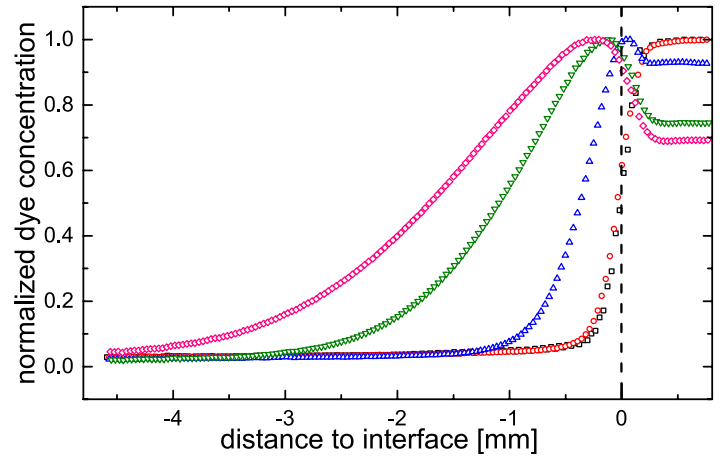

FIG. 7. (a) $\mathrm{D}_{2} \mathrm{O}$ volume fraction and (b) fluorescein concentration (normalized to the maximum) profiles inside and outside the polymer hydrogel at different times after contact with fluorescein solution as determined by neutron radiography and fluorescence imaging, respectively. Symbols correspond to the same times in both figures (as indicated).

i.e., the concentration as a function of the distance to the hydrogel-solution interface (Fig. 7). The temporal evolutions of these profiles provide detailed and quantitative information on the diffusion processes with very good statistics, which can be quantitatively analyzed to yield, e.g., the diffusion coefficients of the solvent(s) and fluorescein molecules in the solution and hydrogel. A quantitative analysis of the concentration profiles is ongoing ${ }^{37}$ and beyond the scope of the present manuscript.

\section{B. Optical reflection imaging: Mesoporous silica contacted with solvent vapor}

This second example investigates the solvent uptake of a nile red-functionalized mesoporous silica (MCM-41) powder which is not transparent to light so that the apparatus had to be used in reflection mode. Functionalized mesoporous silica powders have been proposed as chemical sensors. ${ }^{38}$ Due to their porous structure, these powders exhibit a very large surface area (approximately $1000 \mathrm{~m}^{2} / \mathrm{g}$ ). ${ }^{39}$ While the pores in the powder particles are mainly responsible for this large surface area, there are also relatively large cavities between the individual particles. Thus, the powder contains intra- as well as interparticle cavities.

In this experiment, MCM-41 powder (Sigma Aldrich) loaded with approximately $9 \mu \mathrm{mol} / \mathrm{g}$ of nile red $^{40}$ was contacted with diethyl ether vapor. The aim of this experiment was to compare the uptake of solvent, using NR, and the corresponding change in fluorescence, using FI, after contact with solvent vapor. Generally, the fluorescence of such powders changes when they are contacted with a solvent. The solvent polarity has a strong effect on both the excitation and in particular the emission wavelengths, i.e., these materials exhibit solvatochromism. By using a color camera, it is possible to identify changes in the fluorescence wavelengths, which are very pronounced in the present system, ${ }^{40}$ and thus differentiate between different solvents absorbed. However, in this exploratory example, the intensity of the green component of the fluorescence was rather weak. Thus, we do not consider this possibility further here.

Nile red is excited at $552 \mathrm{~nm}$, a different wavelength compared to fluorescein. Hence, the following components of the apparatus were substituted for the ones previously used: an LED with a center wavelength of $525 \mathrm{~nm}$, an EX filter that transmits a band of $20 \mathrm{~nm}$ around a center wavelength of $542 \mathrm{~nm}$, a DM that reflects wavelengths below and transmits wavelengths above $568 \mathrm{~nm}$, and an EM filter that transmits a band of $52 \mathrm{~nm}$ around $620 \mathrm{~nm}$. Approximately $100 \mathrm{mg}$ powder was held in one chamber of the sample cell shown in Figure 4. The glass window enabled visual verification of the homogeneity of the powder packing. The experiments were performed at room temperature and atmospheric pressure. To ensure that the time of initial contact with the vapor was known, solvent was injected remotely into the solvent chamber after several images of the dry powder had been collected.

During the experiment, the solvent migrated from top to bottom into the sample. Visual observation of the fluorescence images suggests only small changes in the fluorescence of the sample with time (Figs. 8(c) and 8(d)). Clearer changes can be seen in the neutron transmission images (Figs. 8(a) and 8(b)): Silica powder does not absorb neutrons strongly and appears light gray whereas the wet powder appears darker corresponding to a lower neutron transmission due to the presence of liquid solvent containing hydrogen (H) (Fig. 8(b)). Due to its very low density, solvent vapor is practically transparent to neutrons and hence cannot account for the decrease in transmission.

The neutron transmission images can be quantitatively analyzed in a similar manner to that described in the previous example. For the current samples, solvent migration is homo-

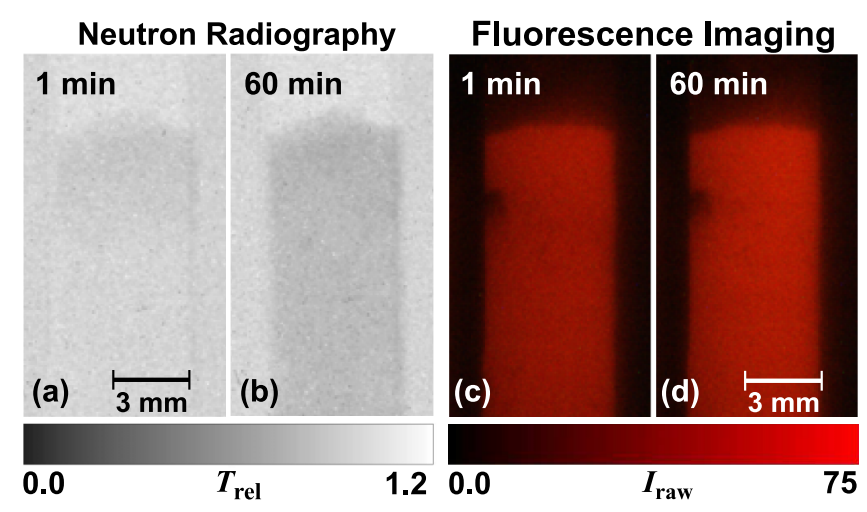

FIG. 8. Neutron radiographs ((a) and (b)) and fluorescence images ((c) and (d)) of MCM-41 powder loaded with $9 \mu \mathrm{mol} / \mathrm{g}$ nile red directly after contact with diethyl ether and $1 \mathrm{hr}$ later. 
geneous, i.e., the solvent concentration is independent of the position in the plane perpendicular to the ingress of solvent (Fig. 8). Hence, the data can be averaged horizontally across the sample to improve statistics. Furthermore, homogeneity in the horizontal plane ensures that the results of reflection imaging are independent of penetration depth and can be compared directly with the neutron imaging results obtained as a projection through the entire sample thickness.

To improve the statistics further, in the analysis, the sample was divided into seven volumes at different heights $h$ in the sample. Edge and surface effects were avoided by binning over an image area $4.05 \mathrm{~mm}$ wide (sample width $5.1 \mathrm{~mm}$ ) by $1.35 \mathrm{~mm}$ high and excluding about $2.4 \mathrm{~mm}$ at the top and several millimeters at the bottom of the sample. The same areas were used in NR and FI.

The neutron transmission $T_{\mathrm{S}}(h, t)$ and the fluorescence intensity $I(h, t)$ were converted to solvent filling factor, $f_{\mathrm{NR}}$, and fractional fluorescence intensity, $f_{\mathrm{FI}}$, respectively, as follows:

$$
\begin{gathered}
f_{\mathrm{NR}}=\frac{T_{\mathrm{S}}(h, t=0)-T_{\mathrm{S}}(h, t)}{T_{\mathrm{S}}(h, t=0)-T_{\mathrm{S}}(h, t \rightarrow \infty)}, \\
f_{\mathrm{FI}}=\frac{I(h, t)-I(h, t=0)}{I(h, t \rightarrow \infty)-I(h, t=0)}
\end{gathered}
$$

and plotted as a function of time (Fig. 9). This assumes linear relationships between solvent content and the NR and FI signals, which is reasonable for the solvent contents considered, and a fully saturated sample at long times, which also appears reasonable given that both, $f_{\mathrm{NR}}$ and $f_{\mathrm{FI}}$, saturate at long times

\section{(a) Neutron Radiography}

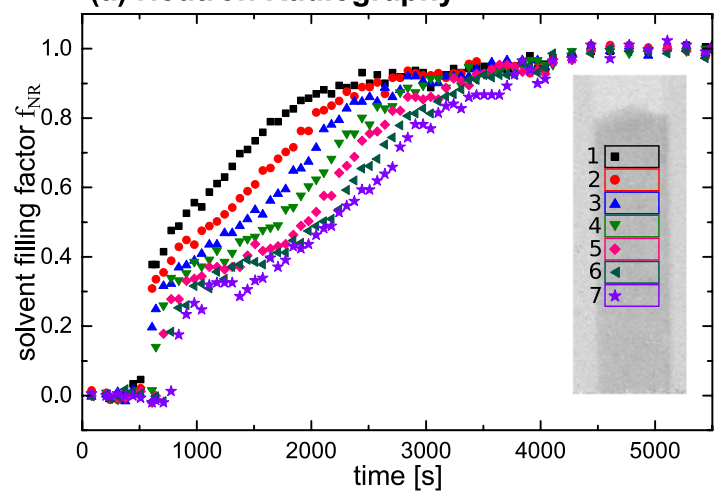

(b) Fluorescence Imaging

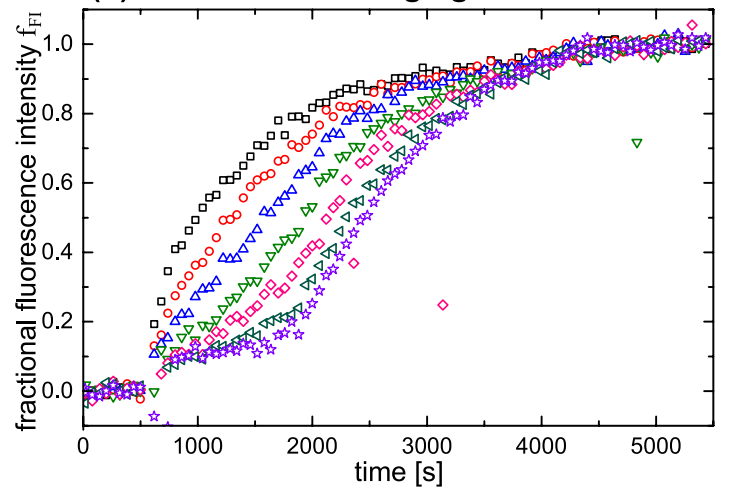

FIG. 9. (a) Solvent filling factor $f_{\mathrm{NR}}$ and (b) fractional fluorescence intensity $f_{\mathrm{FI}}$ in volumes at different positions in the MCM- 41 sample (as indicated in (a)).
(Fig. 9). Since the neutron absorption of vapor is very small, $f_{\mathrm{NR}}$ is a measure of the volume filled with liquid which might be inside or between the powder particles. By contrast, $f_{\mathrm{FI}}$ is sensitive to the amount of the nile red wetted by the solvent. As the nile red is attached to the surfaces, both of the pores and the particles, this quantity reports on the fraction of surface that is wetted.

Both, $f_{\mathrm{NR}}$ and $f_{\mathrm{FI}}$, increase with time with the increase occurring earlier closer to the top of the powder sample (Fig. 9). The increase in $f_{\mathrm{NR}}$ happens in two steps: a fast increase to a value of about 0.3 and a subsequent slower increase to full saturation. While in the first step, mainly $f_{\mathrm{NR}}$ increases, in the second step, $f_{\mathrm{FI}}$ follows $f_{\mathrm{NR}}$ closely (Fig. 10). The delay between the increases in $f_{\mathrm{NR}}$ and $f_{\mathrm{FI}}$ becomes longer as volumes deeper in the sample are considered. This observation suggests that migration of solvent molecules into the free volume occurs in two stages. First, they quickly move into the large cavities between the particles, which, due to their relatively large size, have a large volume-to-surface ratio and thus lead to the significant increase in $f_{\mathrm{NR}}$ but only a modest increase in $f_{\mathrm{FI}}$. Subsequently, in a much slower process solvent moves from these cavities into the smaller pores inside the particles. The pores have a large surface-to-volume ratio and hence both, $f_{\mathrm{NR}}$ and $f_{\mathrm{FI}}$, increase. This is consistent with theoretical considerations, which predict larger cavities to fill more rapidly than small pores. ${ }^{41,42}$ Furthermore, it is conceivable that the total volume of the large cavities represents about one third of the free volume. ${ }^{43}$ The neutron and fluorescence data allow for a more quantitative analysis of the transport process, ${ }^{41,42,44,45}$ which, however, is beyond the scope of this report. Nevertheless, these experiments illustrate that valuable quantitative and complementary information on the migration of solvent into porous systems can be obtained by simultaneously performing neutron and fluorescence imaging, here on the filled volume and wetted surface, respectively. This can then be exploited for the rational design of hierarchically porous systems. ${ }^{46-48}$

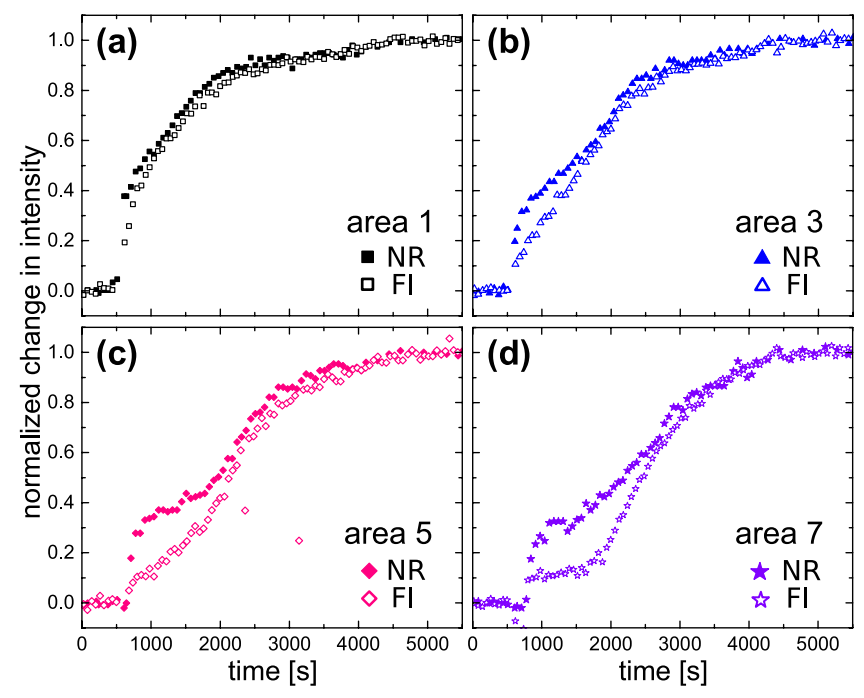

FIG. 10. Direct comparison of the temporal change in $f_{\mathrm{NR}}$ (closed symbols) and $f_{\mathrm{FI}}$ (open symbols) for four selected volumes in the sample. Shape and color of the symbols correspond to the respective sample volumes depicted in Fig. 9. 


\section{SUMMARY AND CONCLUSION}

We have designed and constructed an apparatus which enables neutron radiography to be simultaneously combined with optical and fluorescence imaging. Imaging can be performed in transmission or reflection mode to investigate optically transparent or opaque samples, respectively. The combination with neutron radiography is rendered possible because the optical setup is compact and hence can be incorporated into neutron radiography beamlines. Additionally, the optical components that need to be in the neutron beam do not significantly affect the neutron beam. Therefore, the three techniques can be used simultaneously to obtain complementary and quantitative information on different aspects of the sample and their time dependencies.

To illustrate the operation of the apparatus, two examples were presented. First, the transmission mode was used to investigate diffusion processes following the contact of a polymer hydrogel with dye solution. The three imaging techniques allowed us to follow the diffusion of both solvent and solute (dye), as well as changes in the hydrogel. In the second example, the reflection mode was used to study dyefunctionalized mesoporous silica after it was brought in contact with solvent vapor. Differences in solvent content and wetted surface area were revealed which allowed us to disentangle and distinguish the filling of pores in the particles and cavities between the particles. Extensions of the apparatus, for example, to follow simultaneously two fluorophores or to study solvatochromism, were also indicated.

\section{ACKNOWLEDGMENTS}

We thank the Paul Scherrer Institute (Villigen, Switzerland) for beamtime and the coated mirrors as well as the workshop of the Research Centre Jülich for manufacturing the sample cells. We furthermore acknowledge support by the Strategic Research Fund of the Heinrich Heine University, the International Helmholtz Research School of Biophysics and Soft Matter (IHRS BioSoft) and we thank Jennifer Burbach and Annette Schmidt for assistance with sample preparation and sample advice, respectively.

${ }^{1}$ P. Huber, J. Phys.: Condens. Matter 27, 103102 (2015).

${ }^{2}$ D. W. Schaefer and R. S. Justice, Macromolecules 40, 8501 (2007).

${ }^{3}$ M. Moniruzzaman and K. I. Winey, Macromolecules 39, 5194 (2006).

${ }^{4}$ J. Oberdisse, Soft Matter 2, 29 (2006).

${ }^{5}$ C. A. Dreiss, Soft Matter 3, 956 (2007).

${ }^{6}$ J. Vermant and M. J. Solomon, J. Phys.: Condens. Matter 17, R187 (2005).

${ }^{7}$ P. T. Callaghan, Rep. Prog. Phys. 62, 599 (1999).

${ }^{8}$ S. U. Egelhaaf, U. Olsson, and P. Schurtenberger, Physica B 326, 276 (2000).

${ }^{9}$ S. U. Egelhaaf, U. Olsson, P. Schurtenberger, J. Morris, and H. Wennerström, Phys. Rev. E 60, 5681 (1999).

${ }^{10}$ S. U. Egelhaaf and P. Schurtenberger, Phys. Rev. Lett. 82, 2804 (1999).

${ }^{11}$ A. Onuki, J. Phys.: Condens. Matter 9, 6119 (1997).

${ }^{12}$ R. Mezzenga, P. Schurtenberger, A. Burbidge, and M. Michel, Nat. Mater. 4, 729 (2005).

${ }^{13}$ B. Kainz, E. A. Oprzeska-Zingrebe, and J. L. Herrera, Biotechnol. J. 9, 51 (2013).

${ }^{14}$ G. Sirinakis, Y. Ren, Y. Gao, Z. Xi, and Y. Zhang, Rev. Sci. Instrum. 83, 093708 (2012).

${ }^{15}$ A. R. Kherlopian, T. Song, Q. Duan, M. A. Neimark, M. J. Po, J. K. Gohagan, and A. F. Laine, BMC Syst. Biol. 2, 74 (2008).
${ }^{16}$ D. Ganguly, S. Chakraborty, M. Balitanas, and T.-H. Kim, in SecurityEnriched Urban Computing and Smart Grid, edited by T.-H. Kim, A. Stoica, and R.-S. Chang (Springer Berlin Heidelberg, 2010), Vol. 78, pp. 504-516.

${ }^{17}$ D. B. Murphy and M. W. Davidson, Fundamentals of Light Microscopy and Electronic Imaging, 2nd ed. (John Wiley \& Sons, Inc., New York, 2012).

${ }^{18}$ J. R. Lakowicz, Principles of Fluorescence Spectroscopy (Springer, New York, USA, 2006).

${ }^{19}$ N. Rudolph, H. G. Esser, A. Carminati, A. B. Moradi, A. Hilger, N. Kardjilov, S. Nagl, and S. E. Oswald, J. Soils Sediments 12, 63 (2011).

${ }^{20} \mathrm{P}$. Schurtenberger, in Neutrons, X-Rays and Light: Scattering Methods Applied to Soft Condensed Matter, edited by P. Lindner and T. Zemb (NorthHolland, Elsevier Science B. V., Amsterdam, 2002), pp. 145-170.

${ }^{21}$ R. Hassanein, E. Lehmann, and P. Vontobel, Nucl. Instrum. Methods Phys. Res., Sect. A 542, 353 (2005).

${ }^{22}$ R. Hassanein, F. de Beer, N. Kardjilov, and E. Lehmann, Physica B 385-386, 1194 (2006)

${ }^{23}$ A. S. Tremsin, E. H. Lehmann, N. Kardjilov, M. Strobl, I. Manke, J. B. McPhate, J. V. Vallerga, O. H. W. Siegmund, and W. B. Feller, J. Instrum. 7, C02047 (2012).

${ }^{24}$ U. Matsushima, N. Kardjilov, A. Hilger, I. Manke, H. Shono, and W. Herppich, Nucl. Instrum. Methods Phys. Res., Sect. A 605, 185 (2009).

${ }^{25}$ L. Crow, in Neutron Imaging and Applications: A Reference for the Imaging Community, edited by I. S. Anderson, R. L. McGreevy, and H. Z. Bilheux (Springer, New York, 2009), pp. 47-63.

${ }^{26}$ E. Lehmann, G. Frei, G. Kühne, and P. Boillat, Nucl. Instrum. Methods Phys. Res., Sect. A 576, 389 (2007).

${ }^{27}$ H. E. Hermes, C. E. Sitta, B. Schillinger, H. Löwen, and S. U. Egelhaaf, Phys. Chem. Chem. Phys. 17, 15781 (2015).

${ }^{28}$ H. E. Hermes, R. L. Hanes, D. Wagner, S. Hartmann, A. Kaestner, M. Schulz, B. Schillinger, E. H. Lehmann, and S. U. Egelhaaf, "Using time-resolved neutron imaging to study diffusion and imbibition in soft and complex matter" (unpublished).

${ }^{29} \mathrm{H}$. Li, B. Schillinger, E. Calzada, L. Yinong, and M. Muehlbauer, Nucl. Instrum. Methods Phys. Res., Sect. A 564, 405 (2006).

${ }^{30}$ V. F. Sears, Neutron News 3, 29 (1992).

${ }^{31}$ See http://www.ati.ac.at/ neutropt/scattering/ScatteringLengthsAdvTable. pdf for material dependent values of the total neutron cross sections; accessed 21 June 2015.

${ }^{32}$ N. B. Vicente, J. E. D. Zamboni, J. F. Adur, E. V. Paravani, and V. H. Casco, J. Phys.: Conf. Ser. 90, 012068 (2007).

${ }^{33}$ L. Hodgson, P. Nalbant, F. Shen, and K. Hahn, Methods Enzymol. 406, 140 (2006).

${ }^{34}$ A. Kaestner, S. Hartmann, G. Kühne, G. Frei, C. Grünzweig, L. Josic, F. Schmid, and E. Lehmann, Nucl. Instrum. Methods Phys. Res., Sect. A 659, 387 (2011).

${ }^{35}$ E. Lehmann, A. Kaestner, L. Josic, S. Hartmann, and D. Mannes, Nucl. Instrum. Methods Phys. Res., Sect. A 651, 161 (2011).

${ }^{36}$ R. Messing, N. Frickel, L. Belkoura, R. Strey, H. Rahn, S. Odenbach, and A. M. Schmidt, Macromolecules 44, 2990 (2011).

${ }^{37}$ D. Wagner, J. Burbach, S. U. Egelhaaf, and H. E. Hermes, "Solvent and solute ingress into a cross-linked polymer hydrogel distinguished by a combination of complementary imaging techniques" (unpublished).

${ }^{38}$ B. G. Trewyn, S. Giri, I. I. Slowing, and V. S.-Y. Lin, Chem. Commun. 2007, 3236.

${ }^{39}$ J. S. Beck, J. C. Vartuli, W. J. Roth, M. E. Leonowicz, C. T. Kresge, K. D. Schmitt, C. T. W. Chu, D. H. Olson, and E. W. Sheppard, J. Am. Chem. Soc. 114, 10834 (1992).

${ }^{40} \mathrm{M}$. Börgardts, "Synthesis and optical properties of mesoporous nile redsilica hybrids" (unpublished).

${ }^{41}$ M. Alava, M. Dube, and M. Rost, Adv. Phys. 53, 83 (2004).

${ }^{42}$ S. T. Oyama, M. Yamada, T. Sugawara, A. Takagaki, and R. Kikuchi, J. Jpn. Pet. Inst. 54, 298 (2011).

${ }^{43}$ I. Biazzo, F. Caltagirone, G. Parisi, and F. Zamponi, Phys. Rev. Lett. 102, 195701 (2009).

${ }^{44}$ S. Gruener, Z. Sadjadi, H. E. Hermes, A. V. Kityk, K. Knorr, S. U. Egelhaaf, H. Rieger, and P. Huber, Proc. Natl. Acad. Sci. U. S. A. 109, 10245 (2012).

${ }^{45}$ S. Gruener and P. Huber, Phys. Rev. Lett. 100, 064502 (2008).

${ }^{46}$ P. Innocenzi, L. Malfatti, and G. J. A. A. Soler-Illia, Chem. Mater. 23, 2501 (2011).

${ }^{47}$ C. M. A. Parlett, K. Wilson, and A. F. Lee, Chem. Soc. Rev. 42, 3876 (2013).

${ }^{48}$ N. D. Petkovich and A. Stein, Chem. Soc. Rev. 42, 3721 (2013). 